vertical proportions. American Journal of Orthodontics. 1964;50(11): 801-823. doi:10.1016/ 0002-9416(64) 90039-9

3. Trang NT, Phương NTT, Dũng TM, Hải HV. Đặc điểm mô cứng trên phim so - mặt nghiêng ở Việt trưởng thành có sai khớp cẳn loai I. Accessed September 9, 2021. http:// hocvienquany.edu.vn/ Tapchi_YDHQS/Portal/Default.aspx?MaAbstract $=52257$

4. Ho TTT, Luong QT. Dental-craniofacial Characteristics of Southern Vietnamese People with Well-balanced Face on Cephalometric Films and Its Comparison with Caucasians and Northern Vietnamese Population. J Int Soc Prev Community Dent. 2021;11(3):316-323. doi:10.4103/ jispcd.JISPCD_13_21

5. Kim YH. Overbite depth indicator with particular reference to anterior open-bite. Am J Orthod.
1974;65(6):586-611. doi:10.1016/0002-9416(74) 90255-3

6. Freudenthaler J, Celar A, Kubota M, Akimoto S, Sato S, Schneider B. Comparison of Japanese and European overbite depth indicator and anteroposterior dysplasia indicator values. Eur J Orthod. 2012;34(1):114-118. doi:10.1093/ejo/cjq177

7. MEAW Multi-loop Edgewise Archwire. Dental Library. Published May 14, 2019. Accessed September 6, 2021. https://dental-library.com/ meaw-multi-loop-edgewise-archwire/

8. Enoki $C$, Telles $C$ de $S$, Matsumoto MAN. Dental-skeletal dimensions in growing individuals with variations in the lower facial height. Braz Dent J. 2004;15:68-74. doi:10.1590/ S010364402004000100013

\title{
ĐÁNH GIÁ SỰ CÂN XỨNG MĂT CỦA BÊ̂NH NHÂN SAI KHỚP CẮN LOẠI III TRÊN PHIM CẮT LỚP VI TÍNH 3D
}

\author{
Dương Chí Hiếu ${ }^{1}$, Nguyễn Khánh Long ${ }^{3}$, Nguyễn Trường Minh ${ }^{1,2}$
}

\section{TÓM TẮT}

Giới thiệu: Mục đích của nghiên cứu này là đánh giá sự cân xứng mặt của bênh nhân sai khớp cắn loại III trên phim cắt lớp vi tính 3D. Phương pháp: Nghiên cứu trên phim cắt lớp vi tính 3 chiều của 20 bệnh nhân có sai khớp cắn loại III xương được chia làm 2 nhóm là nhóm bất cân xứng $(n=13)$ và nhóm cân xứng $(n=7)$ theo độ lệch của điểm Menton (Me) mô mềm đến măt phẳng dọc giữa. Kết quả: Bệnh nhân nhóm bất cẩn xứng có độ lệch của điểm Me trên xương và mô mềm lớn hơn có ý nghĩa so với nhóm cân xứng $(p<0,05)$. Trong nhóm bất cân xứng, chiều dài cành lền và thân xương hàm dưới ở bên không lệch lớn hơn bên lệch cùng phía với Me. Độ chênh lệch giữa chiều dài cành lên và thân xương hàm dưới 2 bên trong nhóm bất cân xứng lớn hơn nhóm cân xứng có ý nghĩa thống kê $(p<0,05)$. Khoảng cách từ điểm Gonion và Jugale bên lệch cùng phía với Me đến các mặt phẳng dọc giữa và mặt phằng đứng ngang lớn hởn bên không lếch. Kết luân: Bất cân xứng măt thể hiện chủ yếu taii các vùng thuộc xương hàm dưới ở cành lên và thẩn xương hàm dưới. Trong nhóm bất cân xứng điểm Gonion và Jugale bên không lệch nằm ở phía trước và gần đường giữa hơn bên lệch cho thấy phức hợp xương hàm trên và hàm dưới có xu hướng xoay về cùng phía bên lệch. Do vậy cần đánh giá chính xác sự cân xứng và bất cân xứng để lên kế hoạch phẫu thuật phù hợp.

${ }^{1}$ Viện Đào Tạo Răng Hàm Mặt, Đại học Y Hà Nội

${ }^{2}$ Bệnh viện Đại học Y Hà Nội

${ }^{3}$ Bênh viên Viêt Nam-Cu Ba

Chịu trách nhiêm chính: Nguyễn Trường Minh

Email: nguyentruongminh@hmu.edu.vn

Ngày nhận bài: 24.6.2021

Ngày phản biên khoa họ: 23.8.2021

Ngày duyệt bài: 30.8.2021
Tư khóa: Bất cân xứng mặt, sai khớp cắn loại III, phim cắt lớp vi tính 3D.

\section{SUMMARY \\ ASSESSMENT OF FACIAL SYMMETRY IN SKELETAL CLASS III ON THREE- DIMENSIONAL COMPUTED TOMOGRAPHY}

Introduction: The purpose of this study was to assess facial symmetry in patients with skeletal Class III. Methods: The patients consisted of 20 adults with skeleton class III, divided into the asymmetry group $(n=13)$ and the symmetry group $(n=7)$ according to the degree of soft-menton deviation. Three-dimensional computed tomography scans were obtained with a spiral computed tomography scanner. Landmarks were designated on the reconstructed 3dimensional surface models. Results: In the asymmetry group, patients showed large shift of menton, mandibular ramus and body lengths were significantly longer on the nondeviated side than on the deviated side $(P<0,05)$. The distance between Gonion and Jugale to Mid-Sagital plane and Coronal Plane were longer on the deviated side than on the nondeviated side. Conclusions: Both ramus and body appeared to contribute to mandibular asymmetry. Maxillomandibular complex had roll and yaw rotations to the menton-deviation. Therefore, it is necessary to carefully evaluate these skeletal units when planning a treatment strategy of facial asymmetry.

Keywords: Facial asymmetry, skeleton class III three-dimensional computed tomography.

\section{I. ĐĂTT VẤN ĐỀ}

Sự bất đối xứng trên khuôn mặt thường thấy ở những bệnh nhân bị sai hình xươnng, đặc biệt là những người bi di dang xương loại III ${ }^{1,2}$. Cằm lệch sang một bên là đặc điểm dễ nhận thấy nhất ở những bệnh nhân có khuôn mặt không 
đối xứng, có thể do dịch chuyển vị trí hoặc thay đổi hình thái của cấu trúc hàm dưới ${ }^{3}$. Phim Xquang 2 chiều (2D) thường được sử dụng để đánh giá sự sai hình của xương hàm mă̆t. Tuy nhiên, độ tin cậy của các phép đo trên phim 2D bị hạn chế do sự chồng hình của các cấu trúc quan trọng và khó khăn trong việc xác định các điểm mốc giải phẫu ${ }^{4}$. Hơn nữa, phim chụp 2D không đem lại thông tin một cách chính xác cũng như đầy đủ về khuôn mặt thực tế là các cấu trúc 3D. Hình ảnh chup cắt lớp vi tính ba chiêu (3D) được đề xuất để giải quyết những vấn đề như vậy, vì nó rất quan trọng trong việc chẩn đoán và lập kế hoạch điêu trị cho bệnh nhân bị dị dạng xương hàm mặt ${ }^{5}$. Hwang và cộng sự đã phát hiện ra rằng sự khác biệt về độ nghiêng của cành lên xương hàm dưới che đi sự khác biệt về chiều dài cành lên xương hàm dưới trong các hình ảnh phim chụp Cephalometric và báo cáo rằng hình ảnh $3 \mathrm{D}$ hữu ích trong việc tìm hiểu các cấu trúc không đối xứng trên khuôn mặt ${ }^{4}$. Hiện nay trên thế giới chưa có nhiều nghiên cứu về hình thái bất cân xứng khuôn mặt của bệnh nhân dị dạng xương hàm loại III trên phim 3DCT. Tại Việt Nam cũng chưa có nghiên cứu nào về vấn đề này trên phim 3D-CT. Mục đính của chúng tôi khi thực hiện nghiên cứu này là mô tả một số đặc điểm hình thái cân xứng và bất cân xứng của bệnh nhân dị dạng xương hàm loại III trên phim 3D-CT.

\section{II. ĐỐI TƯỢNG VÀ PHƯƠNG PHÁP NGHIÊN CỨU}

Nghiên cứu được tiến hành trên 20 phim cắt lớp vi tính hàm mặt của các bệnh nhân đến khám tại bệnh viện Đại học Y Hà Nội được chẩn đoán sai khớp cẳn loại III có chỉ định kết hợp chỉnh nha- phẫu thuật trong khoảng thời gian từ
7/2019-7/2021. Các tiêu chí lựa chọn bệnh nhân vào nghiên cứu bao gồm: 1 . Bệnh nhân trưởng thành có sai khớp cắn hạng III do xương có góc $A N B<0^{\circ} ; 2$. Bênh nhân chưa từng điều trị chỉnh nha hay phẫu thuật trước đó. Tiêu chí loại trừ ra khỏi nghiên cứu bao gồm: bệnh nhân rối loạn tâm thần, bệnh nhân không hợp tác, bệnh nhẩn mắc các dị tật bẩm sinh, hội chứng teo nửa mặt hay có tiền sử chấn thương vùng hàm mặt.

Các chỉ số đo lường trong nghiên cứu được thu thập trên phim cắt lớp vi tính hàm mặt được chup bằng máy chup phim CT 128 lát cắt Optima CT660 GE tại bệnh viện Đại học Y Hà Nội và được phân tích bằng phần mềm phân tích $3 D$ chuyên dụng Invivo (Anatomage, San Jose, CA).
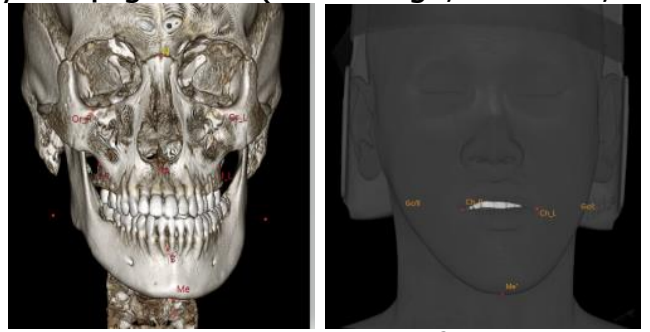

Hình 1. Minh họ mốt số điểm mốc trên mô cứng và mô mè̀m trên phim $3 D^{6}$

Các mặt phẳng tham chiếu: Mặt phẳng dọc giữa (MSP) được xác định đi qua 3 điểm $\mathrm{Na}, \mathrm{Ba}$, $\mathrm{S}$; mặt phẳng ngang Frankfort $(\mathrm{FH})$ đi qua 2 điểm Or, Po bên phải và vuông góc với mặt phẳng dọc giữa; mặt phẳng đứng ngang $(\mathrm{CP})$ đi qua điểm $\mathrm{N}$ và vuông góc với 2 mặt phẳng MSP và $\mathrm{FH}$. Đối tượng nghiên cứu được chia làm 2 nhóm dưa vào độ lệch của $M e^{\prime}$ mô mềm so với mặt phẳng dọc giữa. Nhóm bất cân xứng (nhóm $1)$ : bệnh nhẩn có $M^{\prime}-M S P \geq 3 \mathrm{~mm}$, nhóm cân xứng (nhóm 2): bệnh nhân có $\mathrm{Me}^{\prime}-\mathrm{MSP}<3 \mathrm{~mm}^{4}$.

Bảng 1. Các phép đo trên 3D

\begin{tabular}{|c|c|c|c|}
\hline Kí hiệu & Định nghĩa & Kí hiệu & Định nghĩa \\
\hline Me-S & Khoảng cách từ Me đến MSP & Co-Go & Chiều dài cành lên xương hàm dưới \\
\hline Co-S & Khoảng cách từ Co đến MSP & Go-Me & Chiều dài cành ngang xương hàm dưới \\
\hline Go-S & Khoảng cách từ Go đến MSP & U6MB-S & Khoảng cách từ U6MB đến MSP \\
\hline Go-H & Khoảng cách từ Go đến FH & U6MB-H & Khoảng cách từ U6MB đến FH \\
\hline Go-C & Khoảng cách từ Go đến $\mathrm{CP}$ & $M e^{\prime}-S$ & Khoảng cách từ Me' đến MSP \\
\hline $\mathrm{J}-\mathrm{S}$ & Khoảng cách từ J đến MSP & Ch-H & Khoảng cách từ Ch đến FH \\
\hline $\mathrm{J}-\mathrm{H}$ & Khoảng cách từ ] đến FH & Go'-S & Khoảng cách từ Go' đến MSP \\
\hline
\end{tabular}

\section{KẾT QUẢ NGHIÊN CỨU}

1. Đặc điểm chung của mẫu nghiên cứu. Nghiên cứu có 20 bệnh nhân sai khớp cắn loại III có chỉ định phẫu thuật, trong đó có 10 bệnh nhân nam và 10 bệnh nhân nữ, số bệnh nhân thuộc nhóm bất cân xứng là 13 bệnh nhân, số bệnh nhân thuộc nhóm cân xứng là 7 bệnh nhân.

Bảng 2. Đặc điểm nhóm nghiên cứu

\begin{tabular}{|c|c|c|c|c|}
\hline Giá trị & Bất cân xứng (Nhóm 1) & Cân xứng (Nhóm 2) & Trung bình & $\mathbf{P ( 1 ) ( 2 )}$ \\
\hline $\mathrm{ANB}\left({ }^{\circ}\right)$ & $-3,88 \pm 2,27$ & $-3,37 \pm 2,55$ & $-3,7 \pm 2,32$ & 0,588 \\
\hline
\end{tabular}


VIETNAM MEDICAL JOURNAL N02 - SEPTEMBER - 2021

\begin{tabular}{|c|c|c|c|c|}
\hline ANS-MSP(mm) & $1,47 \pm 0,94$ & $1,06 \pm 0,56$ & $1,32 \pm 0,84$ & 0,135 \\
\hline Me-MSP(mm) & $7,21 \pm 3,62$ & $1,06 \pm 0,58$ & $5,06 \pm 4,18$ & $0,000^{*}$ \\
\hline Me'-MSP(mm) & $7,35 \pm 3,52$ & $1,05 \pm 0,76$ & $5,15 \pm 4,19$ & $0,000^{*}$ \\
\hline
\end{tabular}

*p<0,05 Khác biệt có ý nghĩa thống kê

Khoảng cách từ điểm Me và Me' đến MSP tại nhóm bất cân xứng lớn hơn nhóm cân xứng có ý nghĩa thống kê $(p<0,05)$.

Giá trị ANB trung bình là $-3,7 \pm 2,32\left(^{\circ}\right)$. Khoảng cách từ ANS đến mặt phẳng dọc giữa (MSP) trung bình là 1,32 $\pm 0,84 \mathrm{~mm}$, không có sự khác biệt giữa 2 nhóm ( $p>0,05)$.

2. Sự khác nhau một số chỉ số trên xương của các bệnh nhân sai khớp cắn hạng III do xương

Bảng 3. So sánh số đo trên xương nhóm nghiên cứu

\begin{tabular}{|c|c|c|c|c|c|c|c|}
\hline \multicolumn{3}{|c|}{ Bất cân xứng (Nhóm 1) } & \multicolumn{3}{c|}{ Cân xứng (Nhóm 2) } & \\
\hline Xương & $\begin{array}{c}\text { Bên lệch } \\
(\mathrm{mm})\end{array}$ & $\begin{array}{c}\text { Bên không } \\
\text { lệch }(\mathrm{mm})\end{array}$ & $\begin{array}{c}\text { Khác nhau } \\
(1)(\mathrm{mm})\end{array}$ & $\begin{array}{c}\text { Bên phải } \\
(\mathrm{mm})\end{array}$ & $\begin{array}{c}\text { Bên trái } \\
(\mathrm{mm})\end{array}$ & $\begin{array}{c}\text { Khác nhau } \\
(2)(\mathrm{mm})\end{array}$ & $\begin{array}{c}\mathrm{P} \\
(1,2)\end{array}$ \\
\hline Co-Go & $57,96 \pm 5,54$ & $60,26 \pm 5,19$ & $3,54 \pm 3,08$ & $58,99 \pm 3,34$ & $58,12 \pm 3,38$ & $1,36 \pm 0,85$ & $0,046^{*}$ \\
\hline Go-Me & $87,82 \pm 4,72$ & $89,47 \pm 4,30$ & $3,23 \pm 1,57$ & $88,12 \pm 3,32$ & $88,34 \pm 3,39$ & $1,43 \pm 1,18$ & $0,019^{*}$ \\
\hline Co-S & $51,08 \pm 3,25$ & $51,51 \pm 1,52$ & $2,87 \pm 2,09$ & $51,06 \pm 2,64$ & $50,93 \pm 2,98$ & $1,74 \pm 1,18$ & 0,311 \\
\hline Go-S & $49,42 \pm 3,64$ & $45,16 \pm 2,40$ & $4,57 \pm 2,47$ & $45,91 \pm 5,20$ & $44,85 \pm 3,58$ & $4,35 \pm 2,67$ & 0,938 \\
\hline Go-H & $59,51 \pm 4,65$ & $60,26 \pm 4,34$ & $2,62 \pm 1,98$ & $59,83 \pm 3,62$ & $59,04 \pm 6,02$ & $2,93 \pm 2,08$ & 0,757 \\
\hline Go-C & $69,29 \pm 5,95$ & $65,57 \pm 4,62$ & $3,82 \pm 2,95$ & $64,73 \pm 5,17$ & $64,89 \pm 5,75$ & $1,75 \pm 1,72$ & 0,097 \\
\hline J-S & $32,81 \pm 2,09$ & $29,78 \pm 2,85$ & $3,18 \pm 2,00$ & $32,67 \pm 3,07$ & $31,67 \pm 1,67$ & $2,2 \pm 2,05$ & 0,183 \\
\hline J-H & $28,82 \pm 2,14$ & $28,83 \pm 1,95$ & $1,45 \pm 1,18$ & $29,48 \pm 3,48$ & $29,86 \pm 3,67$ & $1,23 \pm 1,04$ & 0,757 \\
\hline J-C & $23,3 \pm 3,15$ & $21,92 \pm 3,63$ & $2,1 \pm 1,67$ & $21,63 \pm 5,15$ & $21,47 \pm 2,38$ & $2,82 \pm 1,96$ & 0,393 \\
\hline
\end{tabular}

Bên lệch: bên cùng phía với bên lệch Me, Bên không lệch: bên lệch khác phía với Me, Khác nhau $=$ Bên lệch- Bển không lệch, $* p<0,05$ Khác biệt có ý nghĩa thống kê.

Nhóm bất cân xứng có sự khác nhau giữa chiều dài cành lên và thân xương hàm dưới lớn hơn có ý nghĩa so với nhóm cân xứng $(p<0,05)$. Độ dài cành lên xương hàm dưới

của bên lệch $(57,96 \pm 5,54 \mathrm{~mm})$ nhỏ hơn bên không lệch $(60,26 \pm 5,19 \mathrm{~mm})$ và độ dài thân xương hà̀m dưới bên lệch $(87,82 \pm 4,72 \mathrm{~mm})$ cũng nhỏ hơn bên không lệch $(89,47 \pm 4,30 \mathrm{~mm})$. Sự

khác nhau của khoảng cách điểm Gonion và Jugale đến 3 mặt phẳng (MSP, $\mathrm{FH}, \mathrm{CP})$ giữa 2 nhóm cân xứng và không cân xứng không có ý nghĩa $(p>0,05)$. Khoảng cách điểm Gonion và Jugale mặt phẳng dọc giữa và mặt phẳng trán ở bên lệch lớn hơn bển không lệch tại nhóm bất cân xứng. Khoảng cách từ điểm codylion đến mặt phẳng dọc giữa ở hai bên giữa hai nhóm là tương đương nhau.

3. Sự khác nhau một số chỉ số trên răng và mô mềm của các bệnh nhân sai khớp cắn hạng III do xương.

Bảng 4. So sánh số đo trên răng và mô mềm nhóm nghiên cứu

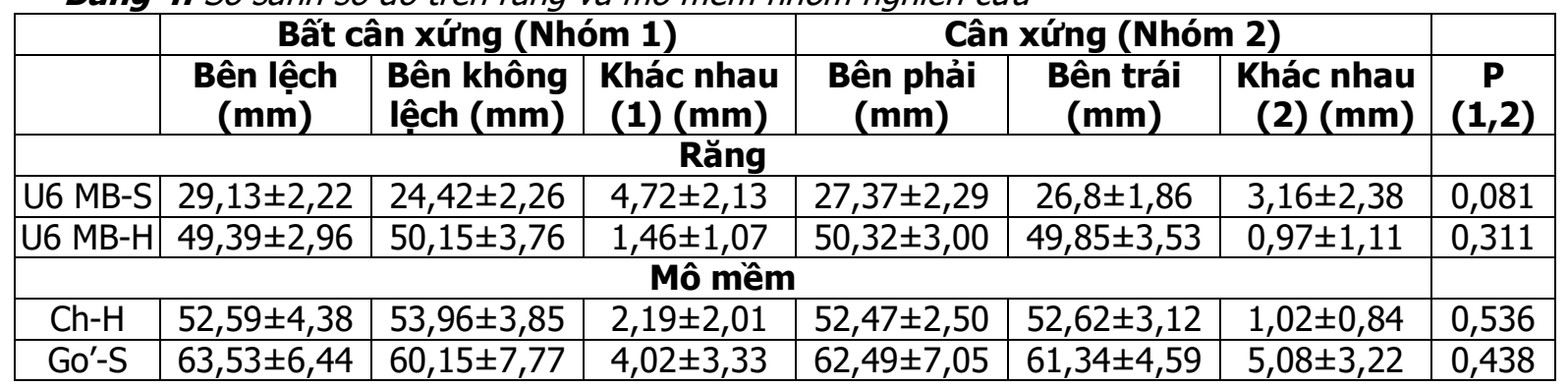

Khoảng cách từ đỉnh múi ngoài gần răng hàm lớn thứ nhất hàm trên đến mặt phẳng dọc giữa ở bên lệch lớn hơn bên không lệch tại nhóm bất cân xứng. Khoảng cách này là tương đương nhau ở nhóm cân xứng. Khoảng cách từ đỉnh múi ngoài gần răng hàm lớn thứ nhất hàm trên đến $\mathrm{FH}$ ở 2 bên của 2 nhóm là tương đương nhau, cho thấy chiều cao của xương hàm trên 2 bên là tương tự nhau. Khoảng cách của điểm khóe môi của 2 nhóm (Chelion) đến mặt phẳng FH ở 2 bên là như nhau. Khoảng cách từ điểm Gonion mô mềm đến mặt phẳng dọc giữa ở nhóm bất cân xứng ở bên lệch lớn hơn bên không lệch tại nhóm bất cân xứng. Khoảng cách này ở nhóm cân xứng là tương đương nhau. Tuy nhiên, sự chênh lệch 2 bên giữa 2 nhóm nghiên 
cứu không có ý nghĩa thống kê ( $p>0,05)$.

\section{BÀN LUÂ̂N}

Trong số 20 bệnh nhân nghiên cứu bênh nhân sai khớp cắn loại III xương có chỉ định phẫu thuật thì số bệnh nhân thuộc nhóm bất cân xứng là 13 cho thấy tỉ lệ bệnh nhân sai khớp cắn loại III tìm đến phương pháp chỉnh nha kết hợp phẫu thuật kèm bất cân xứng về khuôn mặt là khá lớn, tỷ lệ này cũng tương tự như trong nghiên cứu của Kug-Ho You và cộng sự năm $2010^{7}$ có tỉ lệ bệnh nhân thuộc nhóm bất cân xứng chiếm $66,67 \%$ tổng số bệnh nhân nghiên cứu. Khoảng cách Menton đến MSP giữa 2 nhóm bất cân xứng và cân xứng $(7,21 \pm 3,62 \mathrm{~mm}$ và $1,06 \pm 0,58 \mathrm{~mm}$ ) tương tự như trong nghiên cứu của Kug-Ho You và cộng sự năm $2010^{7}$ có khoảng cách của nhóm bất cân xứng và cân xứng lần lượt là $(7,7 \pm 3,4 \mathrm{~mm}$ và $1,2 \pm 0,5 \mathrm{~mm})$. Trong nghiên cứu, nhóm bất cân xứng có độ dài thân xương hàm dưới và cành lên xương hàm dưới ở bên lệch của nhỏ hơn bên không lệch. Điều này tướng tự như trong nghiên cứu của Kug-Ho You và cộng sự năm $2010^{7}$ và HyoYeon Lee và cộng sự năm $2012^{4}$. So sánh với nghiên cứu của Rouh Hwai Wang và cộng sự năm $2020^{8}$ đánh giá các chỉ số xương và mô mềm của 60 người Trung Quốc có khuôn mặt và khớp cắn hài hòa thì nghiên cứu của chúng tôi cho thấy độ dài thân xương hàm dưới tại bên lệch tương đương như trong nghiên cứu của Wang và độ dài thân xương hàm dưới bên không lệch dài hơn trong nghiên cứu của Wang cho thấy quá phát thân xương hàm dưới một bên có thể là một trong các nguyên nhân gây lệch mặt ở nhóm bệnh nhân bất cân xứng.

Trong nhóm bất cân xứng, khoảng cách từ điểm Gonion và Jugale đến MSP và $\mathrm{CP}$ của bên lệch lớn hơn bên đối diện cho thấy điểm Gonion và Jugale bên không lệch nằm ở phía trước và gần đường giữa hơn bên lệch. Điều này cho thấy phức hợp xương hàm trên và hàm dưới có xu hướng xoay về phía bên lệch của nhóm bất cân xứng.

Nghiên cứu các bệnh nhân loại III có chỉ định phẫu thuật chỉnh hình xương hàm là vô cùng quan trọng do đó là cơ sở để có các kế hoạch điều trị phẫu thuật cho bệnh nhân. Trước đây, nghiên cứu chủ yếu dựa trên các phim 2D như cephalometric nên chưa đạt được độ chính xác cũng như có nhiều nguy cơ sai số. Đây là một trong những nghiên cứu đầu tiên ở Việt Nam sử dụng phim 3D để nghiên cứu cấu trúc khuôn mặt ở bệnh nhân loại III xương có chỉ định phẫu thuật.

\section{KẾT LUÂNN}

Nghiên cứu 20 bệnh nhân sai khớp cắn loại III xương đến khám tại bệnh viện Đại học Y Hà Nội có chỉ định kết hợp chỉnh nha- phẫu thuật, sổ bệnh nhân sai khớp cắn loại III do xương có bất cân xứng là 13. Nghiên cứu thực hiên trên phim cắt lớp vi tính 3 chiều cho ta thấy đánh giá tổng quan và đầy đủ hơn đặc điểm khuôn mặt của các bệnh nhân mà khi chụp phim 2 chiều không thấy rõ được. Bệnh nhân nhóm bất cân xứng có độ lệch của điểm Me trên xương và mô mềm lớn hơn có ý nghĩa so với nhóm cân xứng. Trong nhóm bất cân xứng, chiều dài cành lên và thân xương hàm dưới ở bên không lệch lớn hơn bên lệch cùng phía với Me. Nghiên cứu cho thây vị trí có sự bất cân xứng mặt thường là các vùng thuộc xương hàm dưới như vùng cằm, vùng cành ngang, cành lên xương hàm dưới. Phức hợp xương hàm trên và hàm dưới có xu hướng xoay về phía bên lệch của những bệnh nhân sai khớp cắn loại III xương kèm bất cân xứng mặt.

\section{TÀI LIẸU THAM KHẢO}

1. Haraguchi S, Iguchi Y, Takada K. Asymmetry of the face in orthodontic patients. Angle Orthod. 2008; 78(3):421-426.

2. Severt TR, Proffit WR. The prevalence of facial asymmetry in the dentofacial deformities population at the University of North Carolina. Int J Adult Orthodon Orthognath Surg. 1997; 12(3):171-176.

3. Chen Y-J, Yao C-C, Chang Z-C và cộng sứ. Characterization of facial asymmetry in skeletal Class III malocclusion and its implications for treatment. Int J Oral Maxillofac Surg. 2019; 48(12):1533-1541.

4. Lee $H$, Bayome $M$, Kim S-H và cộng sự. Mandibular dimensions of subjects with asymmetric skeletal class III malocclusion and normal occlusion compared with cone-beam computed tomography. Am J Orthod Dentofac Orthop Off Publ Am Assoc Orthod Its Const Soc Am Board Orthod. 2012; 142(2):179-185.

5. Minh NT, Nguyên TM, Hùng Đٕ̃̂̃ và cộng sự. Ứng dụng công nghệ số trong phẫu thuật chinh hình xương hàm. Tạp Chí Học Việt Nam. 2021; 498(2).

6. Swennen GRJ, Schutyser FAC, Hausamen JE. Three-Dimensional Cephalometry: A Color Atlas and Manual. Springer-Verlag; 2006.

7. You K-H, Lee K-J, Lee S-H. Three-dimensional computed tomography analysis of mandibular morphology in patients with facial asymmetry and mandibular prognathism. Am J Orthod Dentofac Orthop Off Publ Am Assoc Orthod Its Const Soc Am Board Orthod. 2010; 138(5):540.e1-8.

8. Wang RH, Ho C-T, Lin H-H. Three-dimensional cephalometry for orthognathic planning: Normative data and analyses. J Formos Med Assoc Taiwan Yi Zhi. 2020; 119(1 Pt 2):191-203. 